\title{
Nonlocal compensation of pure phase objects with entangled photons
}

\author{
Simone Ciald:* \\ Dipartimento di Fisica dell'Università degli Studi di Milano, I-20133 Milano, Italia. and \\ INFN, Sezione di Milano, I-20133 Milano, Italia. \\ Davide Brivid \\ Dipartimento di Fisica dell'Università degli Studi di Milano, I-20133 Milano, Italia. \\ Enrico Tesid手 \\ SUPA, Department of Physics, University of Strathclyde, Glasgow G40NG, Scotland, UK and \\ Dipartimento di Fisica dell'Università degli Studi di Milano, I-20133 Milano, Italia. \\ Matteo G. A. Paris \\ Dipartimento di Fisica dell'Università degli Studi di Milano, I-20133 Milano, Italy and \\ CNISM, Udr Milano, I-20133 Milano, Italy
}

(Dated: June 16, 2018)

\begin{abstract}
We suggest and demonstrate a scheme for coherent nonlocal compensation of pure phase objects based on two-photon polarization and momentum entangled states. The insertion of a single phase object on one of the beams reduces the purity of the state and the amount of shared entanglement, whereas the original entanglement can be retrieved by adding a suitable phase object on the other beam. In our setup polarization and momentum entangled states are generated by spontaneous parametric downconversion and then purified using a programmable spatial light modulator, which may be also used to impose arbitrary space dependent phase functions to the beams. As a possible application, we suggest and demonstrate a quantum key distribution protocol based on nonlocal phase compensation.

PACS numbers: 42.30.Va,03.65.Bg,42.65.Lm
\end{abstract}

\section{INTRODUCTION}

In what is usually referred to as ghost imaging the coherent imaging of an object is achieved with incoherent light upon exploiting the spatial correlations between two light beams [1]. The object interacts with one of the beams and an image of the object is built up by scanning the other beam. Ghost imaging may be obtained either with classically correlated beams [2-4], as those obtained by splitting the light from a (pseudo) thermal source, or with entangled beams [5-7], as those obtained by parametric downconversion. In the latter case one may achieve in principle higher visibility.

For objects which modify only the amplitude of light an image may be obtained with a single spatially incoherent beam upon measuring the autocorrelation function in the far field, without the need of ghost imaging. This is no longer possible when the object is also modifying the phase of the beam. In particular, it is of interest to investigate ghost imaging in the extreme case of pure phase objects, i.e. objects altering only the phase information carried by the beam. Phase objects are also of intrinsic interest in quantum information processing, since they

\footnotetext{
*Electronic address: simone.cialdi@mi.infn.it

${ }^{\dagger}$ Electronic address: davide.brivio@unimi.it

‡Electronic address: enrico.tesio@strath.ac.uk

$\S$ Electronic address: matteo.paris@fisica.unimi.it
}

introduce reversible unitary operations.

Ghost imaging of pure phase objects has been extensively analyzed theoretically and experimentally using both classically or quantum correlated beams [2 [11]. Related effects connected with (nonlocal) dispersion cancellation have been investigated as well, both in the temporal and the spatial domains [12 $[17]$. In this paper we suggest and demonstrate experimentally a scheme to achieve coherent nonlocal compensation/superposition of pure phase objects, also paving the way for the reconstruction of the overall phase function imposed to the two beams. Our scheme is based on two-photon polarization and momentum entangled states, which are generated by spontaneous parametric downconversion and purified using a spatial light modulator. The same device is also used to introduce arbitrary phase functions on the two beams, which represent arbitrary phase objects. In our setup the insertion of a single phase object on one of the beams reduces the purity of the state and the amount of shared entanglement, whereas the original entanglement can be retrieved by adding a suitable phase object on the other beam. The image of both single or double phase objects can be thus obtained by scanning the coincidence counts on one of the two beams. As a possible application, we also suggest and demonstrate a protocol for quantum key distribution based on nonlocal phase compensation.

The paper is structured as follows. In the next Section we describe in details our experimental setup and 
the properties of the two-photon entangled states that are generated. In Section [II we analyze in some details phase imaging and nonlocal phase compensation of phase objects. Finally, in Section IV] we suggest a quantum key distribution protocol based on phase compensation and report about its experimental implementation. Section $\nabla$ closes the paper with some concluding remarks.

\section{EXPERIMENTAL SETUP}

In our setup (see Fig. 1) a two-qubit polarization entangled state is produced by type-I downconversion from a couple of crystals (beta-barium borate, BBO) in a non-collinear configuration [18 20]. Pairs of correlated photons are generated, distributed on a broad angular and spectral ranges, which are determined by the crystal length [31]. Upon expanding the transverse momentum conservation condition to the first order, it can be shown that the angular and the spectral degrees of freedom are connected by the relation

$$
\theta^{\prime}=-\theta+\gamma \omega
$$

where $\theta, \theta^{\prime}$ are the signal and idler shifts from the central emission angles $\left(\Theta_{0} \simeq \Theta_{0}^{\prime} \simeq 3^{\circ}\right.$ in our case $), \omega$ is the signal shift from the central frequency of the downconverted beams, and $\gamma$ is a constant depending only on the signal central frequency and angle [26, 30].

The state at the output of the crystals may be written as

$$
\begin{aligned}
|\psi\rangle \propto \iint d \theta d \theta^{\prime} g\left(\theta, \theta^{\prime}\right) \times & \\
& {\left[|H \theta\rangle\left|H \theta^{\prime}\right\rangle+e^{\imath \Phi\left(\theta, \theta^{\prime}\right)}|V \theta\rangle\left|V \theta^{\prime}\right\rangle\right] }
\end{aligned}
$$

where the overall angular distribution

$$
g\left(\theta, \theta^{\prime}\right)=f\left(\theta, \theta^{\prime}\right) T\left[\omega\left(\theta, \theta^{\prime}\right)\right],
$$

contains the angular distribution $f\left(\theta, \theta^{\prime}\right)$ due to the phase-matching conditions and the transmissivity $T[\omega] \equiv$ $T\left[\omega\left(\theta, \theta^{\prime}\right)\right]$ of an interference filter set on the signal arm. The ket $|P \theta\rangle$ denotes a single photon state emitted with polarization $P=H, V$ at angle $\theta\left(\theta^{\prime}\right)$ along the signal (idler) arm, and the integrations ranges from $-\frac{1}{2} \Delta$ to $\frac{1}{2} \Delta, \Delta$ being the angular aperture of two slits placed along the paths of the downconverted beams (see Fig. 1).

Experimentally, some care must be taken in order to spatially superimpose the $|\mathrm{HH}\rangle$ and $|\mathrm{VV}\rangle$ downconversion beams, so that the angular distribution $f\left(\theta, \theta^{\prime}\right)$ is actually the same for the two components of the entangled state. From Eq. (11) one sees that for a narrow spectral width $(\omega \rightarrow 0)$, the angular distribution $g\left(\theta, \theta^{\prime}\right)$ approaches $f(\theta,-\theta)$ and maximal entanglement in momentum is thus achieved. The relative phase in Eq. (2) can be written as

$$
\Phi\left(\theta, \theta^{\prime}\right)=\Phi_{\mathrm{D}}\left(\theta, \theta^{\prime}\right)+\Phi_{\mathrm{PUR}}\left(\theta, \theta^{\prime}\right)+\phi^{(s)}(\theta)+\phi^{(i)}\left(\theta^{\prime}\right) .
$$

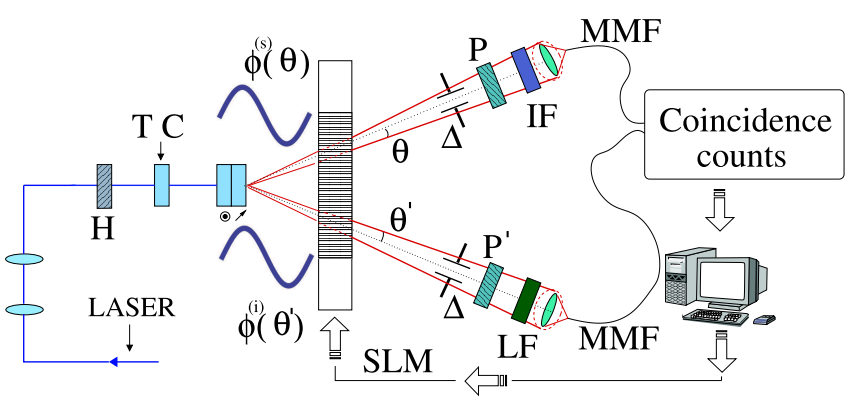

FIG. 1: (Color online) Schematic diagram of experimental setup. A linearly polarized $\mathrm{cw}$ laser diode at $405 \mathrm{~nm}$ pumps a couple of BBO crystals cut for Type-I downconversion. The horizontal and vertical photon pairs are balanced by a half wave-plate set along the pump path, whereas an additional BBO crystal (TC) is placed on the pump path to compensate the temporal delay. Signal and idler beams travel through the SLM, which provides entanglement purification and imposes the space dependent phase functions $\phi^{(s)}(\theta), \phi^{(i)}\left(\theta^{\prime}\right)$, and then are spatially selected by two irises and two slits set at $D=500 \mathrm{~mm}$ with $\Delta x=5 \mathrm{~mm}(\Delta=10 \mathrm{mrad})$. An interference filter IF (FWHM $10 \mathrm{~nm}$ ) set on the signal path selects the spectral width, while a long-pass filter LF, cut-on wavelength $715 \mathrm{~nm}$, is set on the idler path in order to reduce the background. Photons are focused in two multi-mode fibers (MMF) and sent to single-photon counting modules. Polarizers at the angles $\pi / 4$ and $3 \pi / 4$ or $\pi / 4$ and $\pi / 4$ are inserted to measure visibility whereas a quarter-wave plate, a half-wave plate and a polarizer (not shown in the figure) are used for the tomographic reconstruction.

The first phase term, which can be expanded to the first order as

$$
\Phi_{\mathrm{D}}\left(\theta, \theta^{\prime}\right)=\eta\left(\theta-\theta^{\prime}\right)+\Phi_{0},
$$

comes from the angle-dependent optical path of vertically polarized photon pairs, generated in the first crystal, which must travel along the second one. These angular dependent terms are responsible for decoherence of polarization qubit and should be removed in order to obtain an effective source of entangled pairs [25, 26]. In our apparatus, a one dimensional programmable spatial light modulator (SLM) is placed on the signal and the idler paths (see Fig. 1) in order to insert the phase functions $\Phi_{\text {PUR }}\left(\theta, \theta^{\prime}\right), \phi^{(s)}(\theta)$, and $\phi^{(i)}\left(\theta^{\prime}\right)$. The first term is used for purification, i.e to remove the angle-dependent phaseshift, $\Phi_{\mathrm{PUR}}=-\Phi_{\mathrm{D}}$, thus realizing a reliable polarizationentanglement source 26], whereas the functions $\phi^{(s)}$ and $\phi^{(i)}$ represent the phase-objects we insert on the signal and the idler arm, respectively. Notice that spatial light modulators have been recently used in imaging also for a different purpose, i.e that of imposing a set of known random phase distributions [27] and implement the socalled computational ghost imaging [28, 29], where the intensity detected in the one of the beam is computed offline.

Given the state in Eq. (2), we can write the probability for the detection of a photon pair within the emission 
angle range $\theta \in\left(\theta_{0}-\frac{\delta}{2}, \theta_{0}+\frac{\delta}{2}\right), \theta^{\prime} \in\left(\theta_{0}^{\prime}-\frac{\delta^{\prime}}{2}, \theta_{0}^{\prime}+\frac{\delta^{\prime}}{2}\right)$ and with polarization angles $P$ (signal) and $P^{\prime}$ (idler) as follows

$$
\begin{aligned}
C_{\mathrm{PP}^{\prime}}^{\delta \delta^{\prime}}\left(\theta_{0}, \theta_{0}^{\prime}\right)= & \int_{\theta_{0}^{\prime}-\frac{\delta^{\prime}}{2}}^{\theta_{0}^{\prime}+\frac{\delta^{\prime}}{2}} d \theta \int_{\theta_{0}-\frac{\delta}{2}}^{\theta_{0}+\frac{\delta}{2}} d \theta^{\prime} \mid\left\langle P \theta\left|\left\langle P^{\prime} \theta^{\prime} \mid \psi\right\rangle\right|^{2}\right. \\
= & \int_{\theta_{0}^{\prime}-\frac{\delta^{\prime}}{2}}^{\theta_{0}^{\prime}+\frac{\delta^{\prime}}{2}} d \theta^{\prime} \int_{\theta_{0}-\frac{\delta}{2}}^{\theta_{0}+\frac{\delta}{2}} d \theta\left|g\left(\theta, \theta^{\prime}\right)\right|^{2} \\
& \times\left|\cos P \cos P^{\prime}+e^{\imath \Phi\left(\theta, \theta^{\prime}\right)} \sin P \sin P^{\prime}\right|^{2}
\end{aligned}
$$

In order to quantify the entanglement of our state we measure the state visibility, which is defined from Eq. (4) by placing the slits on the SPDC central emission angles, $\theta_{0}, \theta_{0}^{\prime}=0$, and setting the aperture to $\Delta=10 \mathrm{mrad}$, and using the two pairs of angles $P_{1}=P_{1}^{\prime} \equiv \alpha=\pi / 4$ and $P_{2}=\alpha, P_{2}^{\prime}=\alpha+\pi / 2 \equiv \beta$, i.e

$$
V=\frac{C_{\alpha \alpha}^{\Delta \Delta}(0,0)-C_{\alpha \beta}^{\Delta \Delta}(0,0)}{C_{\alpha \alpha}^{\Delta \Delta}(0,0)+C_{\alpha \beta}^{\Delta \Delta}(0,0)} .
$$

In fact, once the state has been purified and the phase objects $\phi^{(s, i)}$ have been inserted, the polarization density matrix reads

$$
\begin{aligned}
\varrho=\frac{1}{2} & \left(|\mathrm{HH}\rangle\left\langle\mathrm{HH}\left|+\varepsilon\left[\phi, \phi^{\prime}\right]\right| \mathrm{VV}\right\rangle\langle\mathrm{HH}|\right. \\
& \left.+\varepsilon^{*}\left[\phi, \phi^{\prime}\right]|\mathrm{HH}\rangle\langle\mathrm{VV}|+| \mathrm{VV}\rangle\langle\mathrm{VV}|\right),
\end{aligned}
$$

where $\varepsilon \equiv \varepsilon\left[\phi, \phi^{\prime}\right]$ is given by

$$
\varepsilon=\int d \theta d \theta^{\prime}\left|g\left(\theta, \theta^{\prime}\right)\right|^{2} \exp \left\{\imath\left[\phi^{(s)}(\theta)+\phi^{(i)}\left(\theta^{\prime}\right)\right]\right\} .
$$

Since the angular distribution $g\left(\theta, \theta^{\prime}\right)$ is symmetric (see below) by choosing, without loss of generality, odd phase functions $\phi^{(s)}$ and $\phi^{(i)}$, we obtain that $\varepsilon$ is real. As a consequence we may write

$$
\varrho=\varepsilon \varrho_{b}+(1-\varepsilon) \varrho_{m},
$$

where $\varrho_{b}$ denotes a Bell state and $\varrho_{m}$ the corresponding mixture. For the state $\varrho$, visibility provides a proper measure of entanglement since the expression in Eq. (5) reduces to $V=\operatorname{Re}[\varepsilon] \equiv \varepsilon$ which, in turn, equals the concurrence of the state.

The experimental setup is shown in Fig. 1) a linearly polarized cw, $405 \mathrm{~nm}$ laser diode (Thorlabs LQC405-40P) pumps a couple of $1 \mathrm{~mm}$ thick BBO crystals cut for TypeI downconversion. The beam waist is set to $\simeq 500 \mu \mathrm{m}$ by a telescopic system. The effective pump power on the generating crystals is of about $11 \mathrm{~mW}$. The $|\mathrm{HH}\rangle$ and $|\mathrm{VV}\rangle$ pairs are balanced by a half wave-plate set along the pump path. A BBO crystal with the proper length and optical axis angle is set on the pump path, and is used to counteract the decoherence effect due to the temporal delay between the two components $2126,30,31$. Such crystal introduces a delay time between the horizontal and vertical polarization of the pump which precompensates the delay time between the $|\mathrm{VV}\rangle$ pair generated by the first crystal and the $|\mathrm{HH}\rangle$ pair from the second one. Signal and idler beams travel through the SLM and are spatially selected by two irises and two slits set at $D=500 \mathrm{~mm}$. The low quantum efficiency of our detectors $(\sim 10 \%)$ forces us to couple large angular regions: we set $\Delta x=5 \mathrm{~mm}$ which corresponds to $\Delta=10 \mathrm{mrad}$. An interference filter IF (FWHM $10 \mathrm{~nm}$ ) is set on the signal arm and selects the spectral width, whereas a long-pass filter set on the idler arm (cut-on wavelength $715 \mathrm{~nm}$ ) is used to reduce the background. Photons are focused in two multi-mode fibers (MMF) and sent to home-made single-photon counting modules, based on an avalanche photodiode operated in Geiger mode with passive quenching. In order to measure the visibility, we insert two polarizers, set at the $P_{1}=P_{1}^{\prime} \equiv \alpha=\pi / 4$, corresponding to a maximum in the coincidence rate, and $P_{2}=\alpha, P_{2}^{\prime}=\alpha+\pi / 2 \equiv \beta$ for the minimum. For the tomographic reconstruction (see below) we insert on both paths a quarter-wave plate, a half-wave plate and a polarizer.

\section{A. Spatial entanglement}

In our setup, the purification provided by the SLM allows us to generate good polarization-entangled states with visibility up to $V=0.912 \pm 0.007$, which may be further increase by spatially filtering the pump to achieve a Gaussian profile. However, ghost imaging also require spatial entanglement and this can be obtained upon exploiting Eq. (11), i.e. by narrowing the output spectral range. We use an interference filter, whose action is denoted by $T[\omega]$ in Eq. (2), which selects a range of about $10 \mathrm{~nm}$ within the overall downconversion spectrum $(\sim 200 \mathrm{~nm})$.

In order to observe the resulting angular correlation we place two slits of aperture $\Delta=1 \mathrm{mrad}$, two polarizers at $P=P^{\prime}=H$ along the downconversion arms and we measure

$$
C \equiv C_{\mathrm{HH}}^{\Delta \Delta}\left(\theta_{0}, \theta_{0}^{\prime}\right)=\int_{\theta_{0}^{\prime}-\frac{\Delta}{2}}^{\theta_{0}^{\prime}+\frac{\Delta}{2}} d \theta^{\prime} \int_{\theta_{0}-\frac{\Delta}{2}}^{\theta_{0}+\frac{\Delta}{2}} d \theta\left|g\left(\theta, \theta^{\prime}\right)\right|^{2},
$$

at $\theta_{0}, \theta_{0}^{\prime}=-2 \Delta,-1.5 \Delta, \ldots,+2 \Delta$. The phase matching (central) angles correspond to $\theta_{0}, \theta_{0}^{\prime}=0$. Coincidences are taken over an acquisition time of $6 \mathrm{~s}$ within a coincidence time window of 50ns. The coincidence counts $C$ are reported in Fig. 2 and the experimental results confirm that $g\left(\theta, \theta^{\prime}\right)$ is approaching $f(\theta,-\theta)$ for the selected (narrow) spectral range. In an analogue way we have also measured $C_{\mathrm{VV}}^{\Delta \Delta}\left(\theta_{0}, \theta_{0}^{\prime}\right)$, checking experimentally that

$$
C_{\mathrm{VV}}^{\Delta \Delta}\left(\theta_{0}, \theta_{0}^{\prime}\right) \simeq C_{\mathrm{HH}}^{\Delta \Delta}\left(\theta_{0}, \theta_{0}^{\prime}\right) .
$$




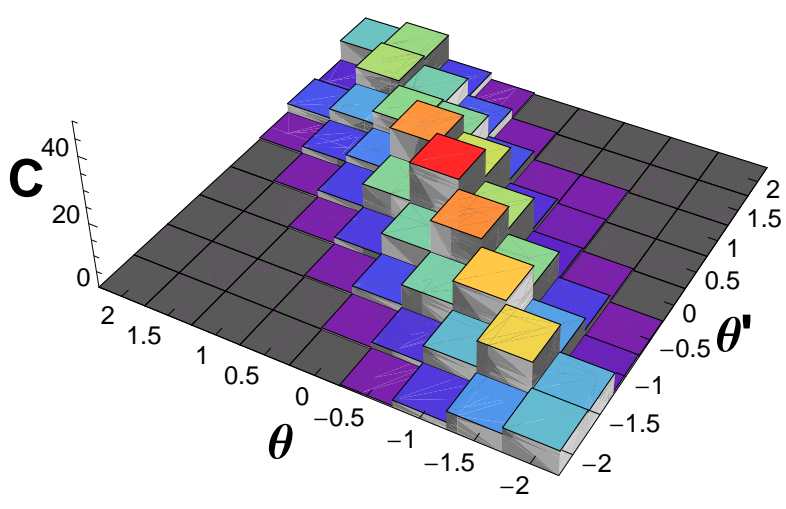

FIG. 2: (Color online) Effect of the interference filter to narrow the output spectral range and achieve spatial entanglement: The plot shows coincidence counts distribution $C$ with $\Delta=1 \mathrm{mrad}$ and polarizers at $P=P^{\prime}=H$ [see Eq. (7)], measured within a coincidence time window of $50 \mathrm{~ns}$, as a function of the signal and idler slit positions $\theta$ and $\theta^{\prime}$. The phase matching central angles correspond to $\theta_{0}, \theta_{0}^{\prime}=0$.

\section{PHASE IMAGING AND NONLOCAL PHASE COMPENSATION}

Single phase-object imaging consists in setting $\phi^{(i)}=0$ and reconstruct the phase function $\phi^{(s)}(\theta)$ inserted along the signal arm by scanning the coincidences for different emission angles $\theta^{\prime}$ on the idler arm [11]. Experimentally, we insert the phase function

$$
\phi^{(s)}(\theta)=a \sin (k \theta)
$$

using the SLM, with $a=1.35 \mathrm{rad}$ and $k \simeq 0.57 \mathrm{mrad}^{-1}$. In Fig. 3 we present the results for the ghost imaging: polarizers are set to $\alpha=\pi / 4$ on the signal and $\beta=3 \pi / 4$ on the idler, the slits aperture are $\Delta=10 \mathrm{mrad}$ for the signal and $\delta=1 \mathrm{mrad}$ for the idler. The signal slit is centered on $\theta_{0}=0$, while the idler slit varies over different values of $\theta_{0}^{\prime}$. The experimental data, already subtracted of the accidental coincidences (coincidence window equal to 50ns, acquisition time 120s ) are the red circles, whereas the red solid line is the theoretical prediction as obtained from Eq. (4), i.e.

$$
\begin{aligned}
C_{\alpha \beta}^{\Delta \delta}\left(0, \theta_{0}^{\prime}\right)=\int_{\theta_{0}^{\prime}-\frac{\delta}{2}}^{\theta_{0}^{\prime}+\frac{\delta}{2}} d \theta^{\prime} \int_{-\frac{\Delta}{2}}^{+\frac{\Delta}{2}} d \theta\left|g\left(\theta, \theta^{\prime}\right)\right|^{2} \\
\times \sin ^{2}\left[\frac{1}{2} \phi^{(s)}(\theta)\right] .
\end{aligned}
$$

For comparison we also report the measured value of the direct counts (blue squares, acquisition time 10s) with the corresponding theoretical prediction (solid blue line), i.e.

$$
C_{\alpha \beta}^{\Delta \delta}\left(0, \theta_{0}^{\prime}\right)=\int_{\theta_{0}^{\prime}-\frac{\delta}{2}}^{\theta_{0}^{\prime}+\frac{\delta}{2}} d \theta^{\prime} \int_{-\frac{\Delta}{2}}^{+\frac{\Delta}{2}} d \theta\left|g\left(\theta, \theta^{\prime}\right)\right|^{2} .
$$

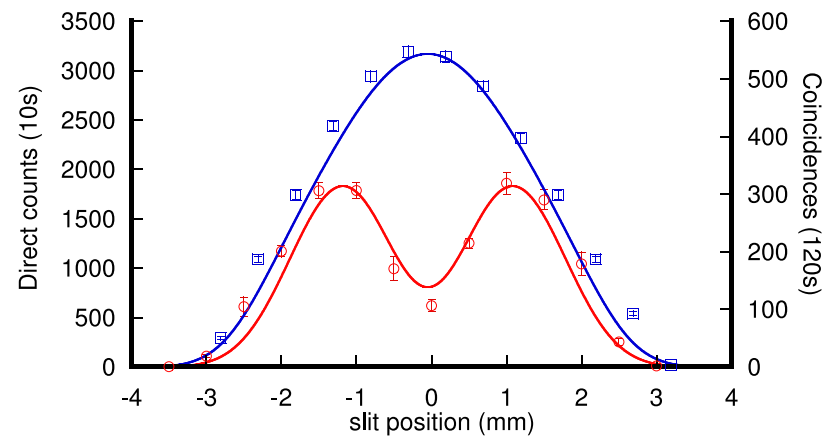

FIG. 3: (Color online) Phase imaging after inserting the phase function $\phi^{(s)}(\theta)=a \sin (k \theta)$ on the signal beam, with $a=1.35 \mathrm{rad}$ and $k \simeq 0.57 \mathrm{mrad}^{-1}$. Blue squares are the direct counts whereas red circles are the coincidences (red circles). Solid lines are the corresponding theoretical predictions (blue and red lines respectively) as a function of the idler slit position. From the coincidence counts observed by scanning the idler beam, one can reconstruct the phase function along the signal beam.

As a matter of fact, the insertion of a single phaseobject leads to the generation of a set of maximally entangled states at different angles, each one with a different phase term $\phi^{(s)}(\theta)$. The entanglement of the state over a broad angular region is thus reduced to $V=0.531 \pm 0.008$.

Once we have reconstructed the signal phase $\phi^{(s)}(\theta)$ we may further tune entanglement by imposing the phase functions $\phi^{(i)}= \pm \phi^{(s)}$ on the idler beam. In this way we nonlocally superimpose two phase object. We remark that, given the correlation condition $\theta^{\prime} \simeq-\theta$ (see Fig. 2), the overall phase function inserted by the SLM is given by

$$
\begin{array}{ll}
\phi^{(s)}(\theta)+\phi^{(i)}\left(\theta^{\prime}\right) \simeq 2 a \sin (k \theta) & \text { if } \quad \phi^{(i)}=-\phi^{(s)} \\
\phi^{(s)}(\theta)+\phi^{(i)}\left(\theta^{\prime}\right) \simeq 0 & \text { if } \quad \phi^{(i)}=\phi^{(s)} .
\end{array}
$$

The corresponding visibility values are $V=0.057 \pm 0.014$ and $V=0.888 \pm 0.003$, respectively. The visibility of the entangled state is sligthly lower than the original value $V=0.912 \pm 0.007$ since the two beams, though showing high angular correlations, are not delta-correlated.

In order to fully characterize the output state, and confirm that visibility is a good figure of merit to discriminate phase functions, we have also performed state reconstruction by polarization qubit tomography for the two different output states. The procedure goes as follows: we measure a suitable set of independent two-qubit projectors 32, 33] and then reconstruct the density matrix from the experimental distributions using maximumlikelihood reconstruction. The tomographic measurements are obtained by inserting a quarter-wave plate, a half-wave plate and a polarizer.

In Fig. 4 we present the tomographic reconstructions of the density matrix of the two output states: the reconstruction of the state resulting from the overall phase function $\phi^{(i)}=\phi^{(s)}$ is reported in the upper panel (real 

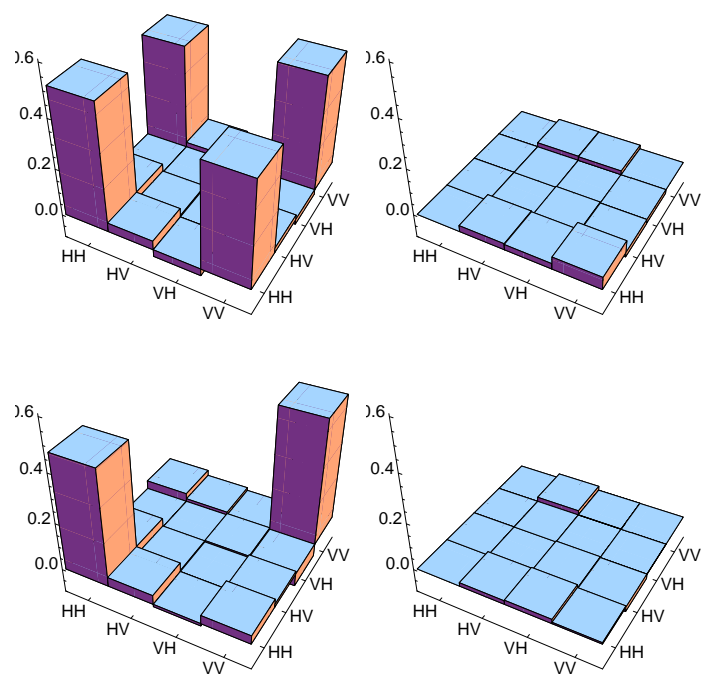

FIG. 4: (Color online) Tomographic reconstruction (the real part of the density matrix on the left and the imaginary one on the right) of the state in which we insert $\phi^{(i)}=\phi^{(s)}=$ $a \sin (k \theta)$, with $a=1.35 \mathrm{rad}$ and $k \simeq 0.57 \mathrm{mrad}^{-1}$ (upper panels) and of the state obtained with $\phi^{(i)}=-\phi^{(s)}$ (lower panels). The corresponding visibilities are given by $0.888 \pm 0.003$ and $0.057 \pm 0.014$, while the measured CHSH-Bell parameters are $B=2.658 \pm 0.011$ and $B=1.854 \pm 0.012$ respectively.

part on the left, and imaginary part on the right). In the lower panel we show the tomographic reconstruction of the density matrix for the state obtained with $\phi^{(i)}=-\phi^{(s)}$ (real part on the left, and imaginary part on the right). In order to detect the presence of nonlocal correlations we also measured the Bell-CHSH parameter

$$
B=\left|E\left(\beta_{1}, \beta_{2}\right)+E\left(\beta_{1}, \beta_{2}^{\prime}\right)+E\left(\beta_{1}^{\prime}, \beta_{2}\right)-E\left(\beta_{1}^{\prime}, \beta_{2}^{\prime}\right)\right|,
$$

where $E\left(\beta_{1}, \beta_{2}\right)$ denotes the correlations between measurements performed at polarization angle $\beta_{j}$ for the mode $j$. We found

$$
\begin{array}{ll}
B=1.854 \pm 0.012 & \text { if } \quad \phi^{(i)}=-\phi^{(s)}, \\
B=2.658 \pm 0.011 & \text { if } \quad \phi^{(i)}=\phi^{(s)},
\end{array}
$$

i.e. we have violation of CHSH-Bell inequality [34 by more than 57 standard deviations for $\phi^{(i)}=\phi^{(s)}$, whereas no violation of the CHSH-Bell inequality is found for $\phi^{(i)}=-\phi^{(s)}$, for which the measures $B$ is less than the threshold $B=2$ by 12 standard deviations.

\section{A QKD PROTOCOL BASED ON NONLOCAL PHASE COMPENSATION}

As a possible application of nonlocal phase compensation, we suggest to exploit the switch between entangled and mixed states for quantum key distribution. Our proposal is based on the fact that Alice and Bob control the signal and the idler arm respectively of the downconversion output. They are thus able to insert independently, and in a random sequence, the phase functions $\phi^{(A)}$ and $\phi^{(B)}$, where $\phi^{(A, B)}= \pm a \sin (k \theta)$. In turn, this may be used to establish a QKD protocol as the analogue of the random choice of the signal basis or of the measurement basis. Alice then encodes the key $(0,1)$ by adding a constant phase $\left(\varphi_{A}=0, \pi\right)$ to $\phi^{(A)}$. Upon setting the detection polarizers to $\alpha=\pi / 4$ and $\beta=3 \pi / 4$ we have that for $\phi^{(B)}=\phi^{(A)}$ a highly entangled state is shared, and thus we have a maximum in the coincidence counting rate when $\varphi_{A}=\pi$ and a minimum when $\varphi_{A}=0$. On the other hand, if $\phi^{(B)}=-\phi^{(A)}$ (or if an eavesdropper tries to acquire knowledge about the key), then Alice and Bob share a mixed (or partially mixed) state, and an intermediate counting rate is detected.

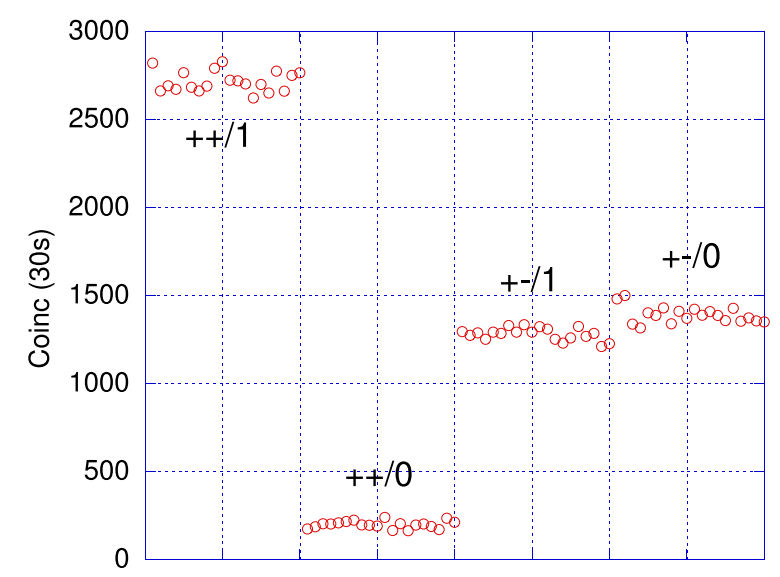

FIG. 5: (Color online) Quantum key distribution by nonlocal phase compensation. The four blocks of data in the plot are the coincidence counting rates in the four different configurations $[++/ 1],[++/ 0],[+-/ 1]$, and $[+-/ 0]$ respectively The first two configurations correspond to the faithful transmission of the key, whereas the two others cases are the events that Alice and Bob are going to reject in the sifting stage of the protocol.

In Fig. 5 we present the experimental results for the coincidence counting rate in the four possible configurations, which are summarized in Table [1] and labelled as follows: $[++/ 1]$ denotes the case $\phi^{(A)}=\phi^{(B)}=a \sin (k \theta)$ and $\varphi_{A}=\pi$, corresponding to the faithful transmission of the symbol "1"; analogously $[++/ 0]$ denotes the case $\phi^{(A)}=\phi^{(B)}=a \sin (k \theta)$ and $\varphi_{A}=0$, leading to the faithful transmission of the symbol " 0 ". The events that Alice and Bob are rejecting in the sifting stage are $[+-/ 0]$ and $[+-/ 1]$ corresponding to $\phi^{(A)}=-\phi^{(B)}=a \sin (k \theta)$ and $\varphi_{A}=0, \pi$ respectively.

Notice that the purification procedure, which removes the angular phase terms $\Phi_{\mathrm{D}}\left(\theta, \theta^{\prime}\right)$, allows us to generate high-quality entanglement states even when broad angular regions are coupled. Indeed, the measurements used for quantum key distribution has been performed with a larger aperture, in order to compensate the low quantum 
TABLE I: A QKD protocol based on nonlocal phase compensation: the phase functions $\phi^{(A)}$ and $\phi^{(B)}$ play the role of the random choice of the signal basis, whereas the key $(0,1)$ is encoded by adding a constant phase $\varphi_{A}=0, \pi$ to $\phi^{(A)}$. When the phase functions are matched, $\phi^{(A)}=\phi^{(B)}$, we have the transmission of the key symbols.

\begin{tabular}{ccccc}
\hline configuration & $\phi^{(A)}$ & $\phi^{(B)}$ & $\varphi_{A}$ & transmitted key \\
\hline$[++/ 1]$ & $a \sin (k \theta)$ & $a \sin (k \theta)$ & $\pi$ & $" 1 "$ \\
{$[++/ 0]$} & $a \sin (k \theta)$ & $a \sin (k \theta)$ & 0 & $" 0 "$ \\
{$[+-/ 1]$} & $a \sin (k \theta)$ & $-a \sin (k \theta)$ & $\pi$ & none \\
{$[+-/ 0]$} & $a \sin (k \theta)$ & $-a \sin (k \theta)$ & 0 & none \\
\hline
\end{tabular}

efficiency of photodetectors and to increase the measurement rate.

\section{CONCLUSIONS}

In conclusion, we have suggested and demonstrated experimentally an entanglement-based scheme to achieve coherent nonlocal compensation of pure phase objects. Our scheme is based on creating two-photon polarization and momentum entangled states where the insertion of a single phase object on one of the beams reduces both the purity of the state and the amount of shared entanglement, and where the original entanglement can be retrieved by adding a suitable phase object on the other beam. In our setup polarization and momentum entangled states are generated by spontaneous parametric downconversion and then purified using a programmable spatial light modulator. The same device is also used to impose arbitrary space dependent phase functions to the beams, which play the role of arbitrary pure phase objects. Finally, we have suggested a novel quantum key distribution protocol exploiting the effect of nonlocal phase compensation and we have provided its experimental verification. Our results prove experimentally the feasiblity of coherent nonlocal compensation/superposition of pure phase objects and pave the way for further developments, as the reconstruction of the overall phase function imposed to the two beams.

\section{ACKNOWLEDGEMENTS}

MGAP thanks Alessandra Gatti and Konrad Banaszek for useful discussions.
[1] A. Gatti, E. Brambilla, and L. A. Lugiato, Progr. Opt. 51, 251 (2008).

[2] R. S. Bennink, S. J. Bentley, and R. W. Boyd, Phys. Rev. Lett. 89, 113601 (2002).

[3] F. Ferri, D. Magatti, A. Gatti, M. Bache, E. Brambilla, and L.A. Lugiato, Phys. Rev. Lett. 94, 183602 (2005).

[4] O. Katz, Y. Bromberg, and Y. Silberberg Appl. Phys. Lett. 95, 131110 (2009).

[5] P. H. Souto Ribeiro, S. Padua, J. C. Machado da Silva, and G. A. Barbosa, Phys. Rev. A 49, 4176 (1994).

[6] T. B. Pittmann, Y. H. Shih, D. V. Strekalov, and S. V. Sergienko, Phys. Rev. A 52, R3429 (1995); D. V. Strekalov, A. V. Sergienko, D. N. Klyshko, and Y. H. Shih, Phys. Rev. Lett. 74, 3600 (1995).

[7] A. F. Abouraddy, B. E. A. Saleh, A. V. Sergienko, and M. C. Teich, Phys. Rev. Lett. 87, 123602 (2001).

[8] Y. T. Zhang, C. J. He, H. G. Li, K. G. Wang, Chin. Phys. Lett. 25, 2481 (2008).

[9] S. Gan, S.H. Zhang, T. Zhao, J. Xiong, X. D. Zhang, K. G. Wang, Appl. Phys. Lett. 98, 111102 (2011)

[10] A. Gatti, E. Brambilla, and L. A. Lugiato, Phys. Rev. Lett. 90, 133603 (2003); A. Gatti, E. Brambilla, M. Bache, and L. A. Lugiato, Phys. Rev. A 70, 013802 (2004).

[11] A. F. Abouraddy, P. R. Stone, A. V. Sergienko, B. E. A. Saleh, and M. C. Teich, Phys. Rev. Lett. 93, 213903 (2004)

[12] A. M. Steinberg, P. G. Kwiat, and R. Y. Chiao, Phys. Rev. Lett. 68, 2421 (1992).

[13] C. Bonato, A. V. Sergienko, B. E. A. Saleh, S. Bonora, P. Villoresi, Phys. Rev. Lett. 101, 233603 (2008).

[14] O. Minaeva, C. Bonato, B. E. A. Saleh, D. S. Simon, A.
V. Sergienko, Phys. Rev. Lett. 102, 100504 (2009).

[15] T. Wasak, P. Szakowski, W. Wasilewski, K. Banaszek Phys. Rev. A 82, 052120 (2010)

[16] J. D. Franson Phys. Rev. A 45, 31263132 (1992).

[17] S-Y. Baek, Y-W. Cho, Y-H. Kim, Opt. Expr. 17, 19241 (2009).

[18] L. Hardy, Phys. Lett. A 161, 326 (1992).

[19] P.G. Kwiat, E. Waks, A. G. White, I. Appelbaum, and P.G. Eberhard, Phys. Rev. A 60, R773 (1999).

[20] M. Genovese, Phys. Rep. 413, 319 (2005).

[21] Y.-H Kim, S. P. Kulik, Y. Shih, Phys. Rev. A 62, 011802 (2000).

[22] Y. Nambu, K. Usami, Y. Tsuda, K. Matsumoto, K. Nakamura, Phys. Rev. A 66, 033816 (2002).

[23] C. Cinelli, G. Di Nepi, F. De Martini, M. Barbieri and P. Mataloni, Phys. Rev. A 70, 022321 (2004); M. Barbieri, C. Cinelli, F. De Martini and P. Mataloni, Las. Phys. 16, 1439 (2006).

[24] G. Brida, M. Genovese, M. V. Chekhova, L. A. Krivitsky, Phys. Rev. A 77, 015805 (2008).

[25] R. Rangarajan, M. Goggin, and P. Kwiat, Opt. Expr. 17, 18920 (2009).

[26] S. Cialdi, D. Brivio, M.G.A. Paris, Appl. Phys. Lett. 97, 041108 (2010).

[27] Y. Bromberg, O. Katz, and Y. Silberberg, Phys. Rev. A 79, 053840 (2009).

[28] J. H. Shapiro, Phys. Rev. A 78, 061802 (2008).

[29] P. Clemente, V. Duràn, V. Torres-Company, E. Tajahuerce, and J.Lancis, Opt, Lett. 35, 2391 (2010).

[30] S. Cialdi, D. Brivio, M. G. A. Paris, Phys. Rev. A 81, 042322 (2010).

[31] S. Cialdi, F. Castelli, I. Boscolo, M. G. A. Paris, Appl. 
Opt. 47, 1832 (2008); S. Cialdi, F. Castelli, M. G. A. Paris, J. Mod. Opt. 56, 215 (2009).

[32] K. Banaszek, G. M. D'Ariano, M. G. A. Paris, M. F. Sacchi, Phys. Rev. A 61 010304(R) (1999).

[33] D. F. V. James, P. G. Kwiat, W. J. Munro, and A. G.
White, Phys. Rev. A 64, 052312 (2001).

[34] J.F. Clauser, M.A. Horne, A. Shimony, and R.A. Holt, Phys. Rev. Lett. 23, 880 (1969). 